Selma Cristina Franco 1

Gastão Wagner de Sousa Campos 1

\footnotetext{
1 Departamento de Medicina Preventiva e Social,

Faculdade de Ciências Médicas, Universidade Estadual de Campinas. Cidade Universitária Zeferino Vaz.

C. P. 6.111 , Campinas, SP 13083-970, Brasil.
}

\section{Avaliação da qualidade de atendimento ambulatorial em pediatria em um hospital universitário}

\author{
Q uality-of-care assessment in a university hospital \\ pediatric clinic
}

Abstract This study focuses on the Pediatric Clinic of the UNICAMP University Hospital, teaching and care facility bel onging to the Brazilian National Health System, providing secondary and tertiary health care for children aged 0 to 18 years. A total of 221 questionnaires were applied with users of the general pediatric out-patient services and one of the specialties, allowing for comparison of various medical care indicators. Users were found to be quite knowledgeable about their health conditions and satisfied with the care received, despite a weak link in the physician-patient relationship. $43.4 \%$ reported problems rel ated to the services, while only $17.6 \%$ made suggestions to improve them. Waiting time was found to be quite long. Users of specialized services differed from general care patients with regard to several indicators.

Key words Quality of Heal th Care; Patient Care Characteristics; Outcome and Process Assessment (Health Care); Health Services

Resumo Estudou-se o ambulatório de Pediatria do Hospital das Clínicas da Unicamp, um serviço di dático-assistencial que, inserido no Sistema Ú ni co de Saúde, presta assistência secundária e terciária para crianças de 0 a 18 anos. Foram apli cados 221 questionários entre clientes do ambulatório geral de pediatria e de uma de suas especiali dades, permitindo comparar diversos indicadores de qualidade da assistência médica prestada. Evidenciou-se um bom nível de conhecimento dos responsáveis pel os usuári os sobre seus problemas de saúde. Encontrou-se al to grau de satisfação dos mesmos, embora houvesse baixíssi ma vinculação entre médico e paciente. Com relação ao atendimento, 43,4\% relataram problemas, mas apenas 17,6\% propuseram sugestões para mel horá-lo. O tempo de espera nas consul tas foi bastantelongo. A clientela da especialidade se diferenciou da do ambulatório geral com relação a diversos indicadores.

Palavras-chave Qualidadedos Cuidados de Saúde; Cuidados Prestados ao Paciente; Avaliação de Processos e Resul tados (Cuidados de Saúde); Servi ços de Saúde 


\section{Introdução}

A avaliação da qualidade dos serviços de saúde é uma preocupação antiga. Atual mente verifica-se uma vigorosa retomada do tema. Relatos demonstram que desde o século passado avaliam-se serviços de modo mais ou menos sistemático (Magalhães, 1983; Ruelas-Barajas \& Vidal-Pineda, 1990). Nas últimas décadas, a maior parte da literatura sobre o assunto tem se concentrado em medições clínicas. A maioria destes trabalhos provém de países desenvolvidos (Gomes, 1989).

Diversos enfoques têm sido dados à avaliação, refletindo as diferentes concepções sobre a qualidade da atenção à saúde. Atualmente reconhece-se essa diversidade conceitual como decorrente das diferentes posições que os atores ocupam no sistema de atenção à saúde, sejam eles os profissionais, as organizações de assistência, os compradores de serviços, os usuários etc (Blumenthal, 1996).

A maioria dos autores situa os objetivos da avaliação dentro do âmbito técnico e racionalizador, ou seja, o de criar normas ou procedimentos, modificar políticas, estabelecer prioridades, visando aumentar a efetividade e a eficácia da atenção, possibilitando a mais adequada al ocação de recursos, segundo critérios técnicos (Horwitz, 1978; De Geyndt, 1986; Escudero, 1989).

Outro objetivo citado é o educativo, propiciando material de ensino para a educação contínua dos profissionais de saúde e definindo a função e responsabilidade do paciente no processo de atenção (De Geyndt, 1986).

Recentemente, em alguns países, têm surgido iniciativas tanto governamentais, quanto da sociedade, exigindo maiores responsabilidades dos prestadores de serviços, com o intuito de proteger os consumidores. O número crescente de leis, normas e regulamentações evidencia este fato (De Geyndt, 1986) e decorre, daí, um outro objetivo da avaliação: reconhecer os direitos de cidadania dos consumidores de serviços de saúde.

Avaliar é, portanto, um passo necessário quando se estabelece a meta de buscar a qualidade na atenção à saúde.

Os métodos propostos para a avaliação estão obviamente relacionados aos seus propósitos e irão influir diretamente em seus resultados (Donabedian, 1978; Gomes, 1989).

Eles têm enfocado aspectos de estrutura, processos ou resultados (Donabedian, 1992), enfatizando ações sobre os indivíduos ou a comunidade, com uma perspectiva institucional (Donabedian, 1988) ou territorial e populacio- nal; centrando-se mais ora nos prestadores (Aguirre-Gas, 1990), ora nos usuários (Velázquez, 1978; Aguirre-Gas, 1990; Jessee, 1990) e ressaltando aspectos clínicos, organizacionais, econômicos ou docentes.

Reconhecendo a crescente importância e legitimidade da perspectiva avaliativa dos usuários sobre a qualidade da atenção, este trabalho pretende avaliar um ambulatório de um hospital universitário, considerando a opinião e satisfação de seus pacientes como indicadores de alguns aspectos dessa qualidade.

\section{Objetivos}

São objetivos desta pesquisa:

1) Analisar alguns indicadores de qualidade da assistência médica prestada no ambulatório de pediatria do Hospital das Clínicas da Unicamp, (HC).

2) Estabelecer comparações entre o ambulatório geral de pediatria e uma especialidade pediátrica (pneumologia/imunologia) quanto a estes indicadores.

3) Verificar, sob a perspectiva dos usuários, os problemas e as soluções propostas para a meIhoria da qualidade da atenção neste ambulatório.

\section{Material e métodos}

\section{Considerações sobre o ambulatório} de pediatria do HC-Unicamp

O presente estudo foi realizado no ambulatório de Pediatria do Hospital das Clínicas da Unicamp, um serviço didático-assistencial do qual participam docentes e médicos contratados do Departamento de Pediatria da Faculdade de Ciências Médicas da Unicamp, além de alunos do quinto ano de graduação em medicina e de residentes de pediatria, de primeiro a terceiro anos.

Inserido no Sistema Ú nico de Saúde, este ambulatório tem como atribuição realizar a atenção secundária e terciária de crianças na faixa etária de 0 a 18 anos, sem delimitação de área de cobertura. Funciona de segunda a sexta-feira e, no mesmo espaço físico, revezam-se os ambulatórios de pediatria clínica (geral) e 12 outras especialidades pediátricas.

No ambulatório geral atende-se amplo espectro de patologias de maior complexidade, refletindo indiretamente o grau de resolutividade da rede de saúde, já que todos os casos encaminhados ao HC passam obrigatoriamen- 
te por ele. O ambulatório de especialidade (pneumologia/imunologia) foi escolhido pelo grande movimento que possui, podendo desta forma representar as especialidades quanto aos aspectos estudados.

No ambulatório geral (também chamado de pediatria clínica), os casos são atendidos indistintamente por alunos e residentes com supervisão de docentes. Os primeiros se revezam neste estágio a cada trinta dias e os últimos a cada dez semanas. Tenta-se fazer com que os retornos sejam atendidos pelo mesmo aluno ou residente que realizou a consulta anterior, na medida em que ele ainda esteja passando pelo setor, o que geralmente é difícil, devido ao longo prazo entre as consultas. No ambulatório de especial idade, o atendimento é realizado por residente de segundo ano, com supervisão de docentes do setor. Aqui também se procura assegurar o seguimento dos casos pelo mesmo residente, o que nem sempre acontece.

Instrumento utilizado para coleta de informações

Entre maio e outubro de 1993, aplicou-se um questionário (Tabela 1) a 221 acompanhantes de pacientes ( 155 do ambulatório geral e 66 da especialidade de pneumologia/imunologia), apenas entre os que compareceram a consultas de retorno, uma vez que as respostas a diversas questões implicavam um conhecimento prévio do funcionamento dos referidos ambulatórios.

Após seleção aleatória e sistemática dos prontuários daquele período, os questionários foram aplicados imediatamente antes das consultas sempre pela mesma entrevistadora, eliminando-se assim problemas relativos a padronizações de perguntas/ respostas. O tamanho das duas amostras foi calculado com base nos dados da média mensal de produção dos referidos ambulatórios no ano de 1992, utilizando-se o calculador estatístico STATCALC do programa EPIINFO versão 5.01b, considerando-se na aplicação de teste de proporções um $p=0,5$ e uma imprecisão de $10 \%$ com probabilidade de $95 \%$. Foram comparadas as diversas variáveis estudadas em cada um dos ambulatórios (geral e especialidade) mediante o teste do Qui-Quadrado, adotando-se o nível de significância de 5\%. Os cálculos foram realizados pelo programa EPIINFO 6.0, através de seu calculador estatístico EPITABLE.

Os resultados foram dispostos em tabelas, construídas pelo programa de análise do EPIINFO 5.01b e editadas pelo MS Word 6.0. Optou-se por não estratificá-los quanto a outras variáveis - sócio-econômica, idade -, por-
Tabela 1

Instrumento para coleta de dados que caracterizem a clientela que freqüenta os ambulatórios de Pediatria do HC Unicamp.

\begin{tabular}{ll}
\hline Entrevista no & Data \\
Idade & Sexo \\
Sabe o que a criança tem? & \\
Diagnóstico referido \\
Melhorou? \\
A que atribui? \\
Q uem é o médico da criança? \\
Sabe os cuidados e orientações em relação à criança?
\end{tabular}

Você está satisfeito com o atendimento neste HC?

Há problemas e falhas no atendimento?

Teria sugestões para melhorar?

Hora de chegada

Minuto de chegada

Hora de saída

Minuto de saída

que o intuito é uma análise geral do conjunto destes indicadores, buscando uma aproximação quanto à qualidade da atenção prestada. A interferência ou não destas variáveis sobre os aspectos avaliados seria objeto de outro trabaIho.

\section{Resultados e discussão}

Os indicadores analisados foram os seguintes:

\section{Conhecimento sobre problema de saúde}

O grau de informação que o paciente possui sobre seu problema de saúde está diretamente relacionado à qualidade da atenção recebida e indica a preocupação do(s) profissional(is) em torná-lo atuante nos diversos momentos de seu tratamento. Quando se perguntou aos acompanhantes sobre o conhecimento a respeito do problema de saúde da criança, foram obtidas as seguintes respostas:

Sim: indicando algum grau de conhecimento sobre a investigação, seguimento ou patologia da criança, permitindo-Ihe situar-se diante do problema. Não era necessário dizer o nome da patologia, mas saber explicar com suas próprias palavras o que a criança tinha ou o motivo pelo qual estava em acompanhamento no HC.

Não: indicando total desconhecimento a respeito do problema de saúde, isto é, respon- 
dendo vagamente à questão formulada, sem situar-se objetivamente quanto ao problema.

Encontrou-se uma porcentagem alta de acompanhantes de pacientes com algum grau de conhecimento sobre a condição de saúde das crianças $(94,1 \%)$, sendo a diferença entre os ambulatórios geral e de especialidade sem significância estatística.

Conhecimento sobre os cuidados em relação à patologia das crianças

Também evidencia o grau de informação dos usuários. As respostas obtidas foram assim codificadas:

Só urgências: soube apenas relatar as orientações para situações de urgência e não no seguimento rotineiro da criança.

Não conhece: não soube relatar nenhum cuidado.

Razoável: quando relatou até três orientações.

Conhece: soube informar pelo menos quatro orientações recebi das pelos profissionais.

Não há: não havia nenhum cuidado recomendado até o momento (Tabela 2).

Vê-se que apenas 3,6\% dos acompanhantes desconhecem as orientações médicas com relação à patologia da criança, sem muita variação entre os dois ambulatórios.

Há, entretanto, grande variação na resposta 'não há orientações', provavelmente em decorrência da diferença do tipo de patologia atendido nos dois ambulatórios, uma vez que a especialidade lida, de maneira geral, com patologias crônicas, cujo manejo implica diversas orientações. Há, também, bastante diferença entre os que sabem claramente as orientações, variando de $59,4 \%$ a $78,8 \%$ ( $p=0,005)$, levando-nos a concluir que a clientela do ambulatório de especialidade é mais bem orientada so- bre os cuidados que devem ser dispensados à sua patologia.

Os dois indicadores apontados acima referem-se ao grau de informação que o paciente recebe durante seus contatos com os médicos, constituindo um aspecto importante da própria relação médico-paciente (Gomes, 1989; Campero-Cuenca, 1990).

Os resultados obtidos demonstram que a clientela usuária do ambulatório de Pediatria tem informações adequadas sobre seu problema de saúde, permitindo-Ihes assumir um papel ativo no seu tratamento. De fato, isto é esperado em um hospital universitário, onde a formação médica necessita privilegiar este aspecto da relação médico-paciente.

\section{Evolução clínica do paciente}

Enfocada a partir da própria ótica do paciente, as respostas encontradas foram (Tabela 3):

Uma porcentagem bastante alta de acompanhantes dos pacientes (71\%) refere melhora clínica desde que estes iniciaram seguimento no HC. Ao compararmos os dois ambulatórios, verificamos diferença estatisticamente significativa entre eles.

Fatores relacionados à evolução clínica anteriormente relatada

Também indagados sob a perspectiva do usuário. As respostas obtidas foram assim codificadas:

Remédios: refere-se ao uso de medicamentos, imunoterapia e combinações com outras categorias acima (com exceção das orientações médicas, que são uma categoria distinta).

Orientações médicas: refere-se às orientações fornecidas pelos médicos e combinações com outras categorias acima (exceto remédios, também uma categoria distinta).

Tabela 2

Distribuição da clientela quanto ao conhecimento sobre os cuidados com a criança.

\begin{tabular}{|c|c|c|c|c|c|c|}
\hline \multirow[t]{2}{*}{ Conhecimento dos cuidados } & \multicolumn{2}{|c|}{ Total } & \multicolumn{2}{|c|}{ Ambulatório geral } & \multicolumn{2}{|c|}{ Ambulatório de especialidade } \\
\hline & $\mathrm{n}$ & $\%$ & $\mathrm{n}$ & $\%$ & $\mathrm{n}$ & $\%$ \\
\hline Só urgências & 2 & 0,9 & 2 & 1,3 & - & - \\
\hline $\mathrm{Não}$ conhece & 8 & 3,6 & 6 & 3,9 & 2 & 3,0 \\
\hline Razoável & 23 & 10,4 & 12 & 7,7 & 11 & 16,7 \\
\hline Não há & 44 & 19,9 & 43 & 27,7 & 1 & 1,5 \\
\hline Conhece & 144 & 65,2 & 92 & 59,4 & 52 & 78,8 \\
\hline Total & 221 & 100,0 & 155 & 100,0 & 66 & 100,0 \\
\hline
\end{tabular}

Nota: A análise estatística, comparando os dois ambulatórios, mostra os seguintes valores de $p$ :

Não conhece: $p=0,850$; Razoável: $p=0,046$; Não há: $p=0,000008$; Conhece: $p=0,005$. 
Remédios e orientações médicas: combinando as duas respostas.

Investigando: quando não havia uma resposta terapêutica até aquele momento por estar sob investigação diagnóstica.

Espontânea: quando a evolução (melhora/ piora) ocorreu sem qualquer tipo de intervenção, por exemplo, devido à mudança climática, ao crescimento da criança etc.

Procedimento cirúrgico: quando considerado o fator mais importante, mesmo que combinado com outros.

Outras práticas: inclui benzedeiras, remédios caseiros, suspensão de tratamento anterior, fisioterapia.

Psicoterapia: quando exclusiva ou sendo o fator mais importante.

Desconhece: quando não soube explicar a evolução (melhora/piora).
Não procede: quando a evolução do caso é por si mesma lenta, como, por exemplo, baixa estatura.

Má condução: toda vez que a condução do caso não esteve de acordo com a expectativa do paciente, como dificuldade no acesso a especialistas e a imunoterapia, morosidade na condução etc (Tabela 4).

Observa-se que a maioria dos responsáveis pelos pacientes relaciona sua evolução clínica ao uso de remédios (24\%), seguindo-se o uso de remédios combinado com orientações médicas (19\%). Estas relações se evidenciam mais ainda no ambulatório de especialidade, no qual estes dois itens aparecem em $65,2 \%$ das respostas.

No ambulatório geral, ocorre o inverso, isto é, o uso de remédios e remédios mais orientações médicas são respostas menos freqüentes

\section{Tabela 3}

Distribuição da clientela quanto à evolução clínica.

\begin{tabular}{|c|c|c|c|c|c|c|}
\hline \multirow[t]{2}{*}{ Evolução clínica } & \multicolumn{2}{|c|}{ Total } & \multicolumn{2}{|c|}{ Ambulatório geral } & \multicolumn{2}{|c|}{ Ambulatório de especialidade } \\
\hline & $\mathrm{n}$ & $\%$ & $\mathrm{n}$ & $\%$ & $n$ & $\%$ \\
\hline Piorou & 5 & 2,3 & 3 & 1,9 & 2 & 3,0 \\
\hline Não melhorou & 59 & 26,7 & 49 & 31,6 & 10 & 15,2 \\
\hline Melhorou & 157 & 71,0 & 103 & 66,5 & 54 & 81,8 \\
\hline Total & 221 & 100,0 & 155 & 100,0 & 66 & 100,0 \\
\hline
\end{tabular}

Nota: A análise estatística, comparando os dois ambulatórios, mostra os seguintes valores de $p$ :

Piorou: $p=0,939$; Não melhorou: $p=0,011$; Melhorou: $p=0,021$.

Distribuição da clientela quanto ao fator relacionado à evolução.

\begin{tabular}{|c|c|c|c|c|c|c|}
\hline \multirow{2}{*}{ Fator relacionado à evolução } & \multicolumn{2}{|c|}{ Total } & \multicolumn{2}{|c|}{ Ambulatório geral } & \multicolumn{2}{|c|}{ Ambulatório de especialidade } \\
\hline & $\mathrm{n}$ & $\%$ & $\mathrm{n}$ & $\%$ & $\mathrm{n}$ & $\%$ \\
\hline Remédios & 53 & 24,0 & 28 & 18,1 & 25 & 37,9 \\
\hline Remédios/O rientações Médicas & 42 & 19,0 & 24 & 15,5 & 18 & 27,3 \\
\hline Investigando & 38 & 17,2 & 32 & 20,6 & 6 & 9,1 \\
\hline O rientações M édicas & 23 & 10,4 & 19 & 12,3 & 4 & 6,1 \\
\hline Espontânea & 23 & 10,4 & 18 & 11,6 & 5 & 7,6 \\
\hline Desconhece & 11 & 5,0 & 11 & 7,1 & - & - \\
\hline Má condução & 11 & 5,0 & 6 & 3,9 & 5 & 7,6 \\
\hline Não procede & 8 & 3,6 & 8 & 5,2 & - & - \\
\hline Outro & 7 & 3,2 & 6 & 3,9 & 1 & 1,5 \\
\hline Cirurgia & 3 & 1,4 & 2 & 1,3 & 1 & 1,5 \\
\hline Psicoterapia & 2 & 0,9 & 1 & 0,6 & 1 & 1,5 \\
\hline Total & 221 & 100,0 & 155 & 100,0 & 66 & 100,0 \\
\hline
\end{tabular}

Nota: A análise estatística, comparando os dois ambulatórios, mostra os seguintes valores de $p$ :

Remédios: $p=0,001$; Rem./O rient. méd.: $p=0,040$; Investigando: $p=0,037 ; 0$ rient. méd.: $p=0,167$; Espontânea:

$p=0,368$; Desconhece: $p=0,045 ;$ Má condução: $p=0,343 ; 0$ utro: $p=0,607$; Cirurgia: $p=$ ?; Psicoterapia: $p=$ ? 
(sendo a diferença significativa estatisticamente). Embora as respostas 'espontânea', 'orientações médicas exclusivas', 'investigando' e 'desconhecida' aumentem, somente as duas últimas são significativamente diferentes quando comparadas ao ambulatório de especialidade.

Em mais da metade dos casos, atribuiu-se a melhora apenas aos itens remédios e orientações médicas, demonstrando a expectativa dos responsáveis pelos pacientes centrada nestes dois itens. De fato, o ambulatório de Pediatria de um hospital terciário, ao lidar com patologias teoricamente de maior complexidade ou gravidade, deveria real mente utilizar-se tanto de medicamentos, quanto de orientações médicas no tratamento das mesmas.

Um fato que nos causou certa estranheza foi a maior incidência da resposta 'má condução do caso' no ambulatório de especialidade, quando comparada à sua incidência no ambulatório geral. Isto provavelmente se deve ao maior nível de exigência da clientela deste ambulatório, ou talvez à expectativa de receber um cuidado específico, como imunoterapia para pacientes portadores de asma brônquica.

Ao se cruzarem os resultados destes dois últimos indicadores, nota-se que a evolução favorável continua a ser atribuída ao uso de remédios associados ou não às orientações médicas (60\%), e a desfavorável deve-se à investigação ainda incompleta e à má condução (78\%).

Estabelecimento de vínculo entre o paciente e o médico que o acompanha

Ao se perguntar sobre quem era o médico responsável pelo acompanhamento da criança, foram obtidas as seguintes informações:

Um: um único profissional identificado nominalmente pelo usuário.
Desconhece: quando não soube identificar o responsável pelo seguimento da criança.

Vários: relatou que era atendido por profissionais diversos, porém sem conseguir identificar qualquer um deles, dando apenas descrições vagas (louro alto).

Vários/citando um ou mais: afirmou que eram vários profissionais, porém identificava nominalmente um ou mais deles (Tabela 5).

Nota-se que $14,5 \%$ dos responsáveis pelos pacientes não sabem quem é o médico encarregado do caso e 42,5\% apontam vários. Assim, do ponto de vista de estabelecimento de vínculo com o profissional que acompanha o caso, pode-se dizer que $57 \%$ dos acompanhantes de pacientes seguidos no ambulatório de Pediatria não conseguem identificar o responsável pelo acompanhamento do filho. Em 8,6\%, há um único profissional envolvido no seguimento do paciente e, em 34,4\%, há vários, porém identificados pelo paciente ou acompanhante.

A comparação entre os dois ambulatórios mostra que a inexistência de vínculo varia de $65,2 \%$ no geral para $37,9 \%$ na especialidade $(p=0,0001)$.

No ambulatório de especialidade, 54,6\% dos casos são seguidos por vários médicos, porém todos conhecidos pelo paciente, mostrando um trabal ho de equipe com estabelecimento de vínculo, diferentemente do que ocorre no ambulatório geral, em que o trabalho de uma equipe reconhecida pelo paciente se dá em $25,8 \%$ do casos ( $p=0,00003$ ).

Diversos autores, ao abordarem este indicador de qualidade na literatura, têm enfocado a satisfação dos pacientes não em termos do ato médico em si, mas, sim, de aspectos relacionados com o trato humanitário, a relação médico-paciente, a informação, entre outros (Aguirre-Gas, 1990; Osborne \& Thompson, 1975; Donabedian, 1979; Kumate $\&$ Isibasis, 1986).

Tabela 5

Distribuição da clientela quanto ao médico responsável pelo caso.

\begin{tabular}{|c|c|c|c|c|c|c|}
\hline \multirow[t]{2}{*}{ Médico responsável } & \multicolumn{2}{|c|}{ Total } & \multicolumn{2}{|c|}{ Ambulatório geral } & \multicolumn{2}{|c|}{ A mbulatório de especialidade } \\
\hline & $\mathrm{n}$ & $\%$ & $\mathrm{n}$ & $\%$ & $\mathrm{n}$ & $\%$ \\
\hline Um & 19 & 8,6 & 14 & 9,0 & 5 & 7,6 \\
\hline Desconhece & 32 & 14,5 & 26 & 16,8 & 6 & 9,1 \\
\hline Vários/Cita 1 & 38 & 17,2 & 20 & 12,9 & 18 & 27,3 \\
\hline Vários/Cita 2 ou + & 38 & 17,2 & 20 & 12,9 & 18 & 27,3 \\
\hline Vários & 94 & 42,5 & 75 & 48,4 & 19 & 28,8 \\
\hline Total & 221 & 100,0 & 155 & 100,0 & 66 & 100,0 \\
\hline
\end{tabular}

Nota: A análise estatística, comparando os dois ambulatórios, mostra os seguintes valores de $p$ :

Desconhece: $p=0,137 ;$ Um: $p=0,723$; Vários/cita um: $p=0,009 ;$ Vários/cita dois ou mais: $p=0,009 ;$ Vários: $p=0,006$ 
Para Campos (1992), o vínculo é um dos elementos essenciais para a instituição de uma prática clínica de qualidade, permitindo tanto aumentar a eficácia das ações de saúde, como introduzir no espaço dos serviços públicos uma das formas de controle social mais efetivas.

A falta de vinculação entre médicos e usuários do ambulatório geral de Pediatria mostranos ser este, talvez, o maior problema assistencial deste ambulatório, retratando uma situação em que, nos serviços públicos de saúde, a relação médico-paciente é impessoal e o paciente se vincula ao serviço e não a pessoas. Embora a assistência neste ambulatório envolva um grande número de pessoas (alunos, residentes, médicos contratados e docentes), com rodízios variados, é necessário reverter-se esta situação, criando mecanismos que garantam ao paciente a possibilidade de se vincular a pessoas que sejam responsáveis por todo o seu seguimento no $\mathrm{HC}$.

Neste sentido, Campos (1992) propõe que o vínculo doente-família ocorra não apenas com um único profissional, mas com uma equipe, ficando bem caracterizado de quem é a responsabilidade de cada tarefa, em situações de rotina ou imprevistas. Achamos que esta proposta é bastante viável no ambulatório de pediatria, inclusive fixando-se dias em que o paciente já saiba que vai ter a oportunidade de chegar ao(s) seu(s) médico(s). O agendamento centralizado num setor específico e controlado por computador precisa ser revisto, para que se possa incorporar esta nova possibilidade.

\section{Satisfação no atendimento}

\section{Categorizado conforme mostra a Tabela 6:}

Existe, de forma geral, um grau muito bom de satisfação relatado pelos responsáveis dos pacientes (93,2\% de usuários satisfeitos), sem diferença estatística nas distinções encontradas entre os dois ambulatórios. Na especialidade, não há pacientes insatisfeitos, apenas 1,5\% de mais ou menos satisfeitos, o que não é significativamente diferente dos $5,8 \%$ do ambulatório geral ( $p=0,238)$.

A satisfação dos usuários de serviços de saúde tem sido enfocada cada vez mais na literatura (Velázquez, 1978; Morera, 1981; Sommers, 1983; Aguirre-Gas, 1990; Chàvez et al., 1990; Jessee, 1990; Donabedian, 1992; Nogueira, 1994), como indicador de qualidade da atenção.

A discrepância encontrada entre os muitos problemas relatados e o alto grau de satisfação dos usuários do ambulatório de Pediatria poderia ser explicada pela limitação da própria metodologia utilizada na coleta dos dados (Thiollent, 1987), o questionário, já que a forma de abordagem dos entrevistados geralmente induz a um tipo de resposta (o 'sim', por exemplo, tende a ser favorecido) ou, então, pelo fato de que os problemas enfrentados não são relevantes para os usuários quando comparados seja com a qualidade da atenção recebida, seja com sua falta de opção de serviço de saúde a ser utilizado.

\section{Tempo de espera}

Foi o tempo contado desde a chegada do paciente ao $\mathrm{HC}$ até o término de sua consulta na Pediatria (Tabela 7).

Verifica-se que o tempo de espera no ambulatório de Pediatria distribui-se mais ou menos igualmente entre as faixas acima. A média de tempo de espera foi de três horas e 36 minutos e a mediana, de três horas e meia. Embora não seja possível visualizar nas tabelas, gostaríamos de ressaltar ter havido 13 pacientes que esperaram sete horas ou mais para serem atendidos!! Está justificado o nome que designa as

Tabela 6

Distribuição da clientela quanto à satisfação no atendimento.

\begin{tabular}{|c|c|c|c|c|c|c|}
\hline \multirow[t]{2}{*}{ Satisfação da clientela } & \multicolumn{2}{|c|}{ Total } & \multicolumn{2}{|c|}{ Ambulatório geral } & \multicolumn{2}{|c|}{ Ambulatório de especialidade } \\
\hline & $\mathrm{n}$ & $\%$ & $\mathrm{n}$ & $\%$ & $n$ & $\%$ \\
\hline Não & 5 & 2,3 & 5 & 3,2 & - & - \\
\hline Mais ou menos & 10 & 4,5 & 9 & 5,8 & 1 & 1,5 \\
\hline $\operatorname{Sim}$ & 206 & 93,2 & 141 & 91,0 & 65 & 98,5 \\
\hline Total & 221 & 100,0 & 155 & 100,0 & 66 & 100,0 \\
\hline
\end{tabular}

Nota: A análise estatística, comparando os dois ambulatórios, mostra os seguintes valores de $p$ :

Mais ou menos: $p=0,238$; Sim: $p=0,064$. 
Tabela 7

Distribuição da clientela quanto ao tempo de espera.

\begin{tabular}{|c|c|c|c|c|c|c|}
\hline \multirow[t]{2}{*}{ Tempo de espera } & \multicolumn{2}{|c|}{ Total } & \multicolumn{2}{|c|}{ Ambulatório geral } & \multicolumn{2}{|c|}{ Ambulatório de especialidade } \\
\hline & $\mathrm{n}$ & $\%$ & $\mathrm{n}$ & $\%$ & $n$ & $\%$ \\
\hline Até $2 \mathrm{~h} 30 \mathrm{~min}$ & 58 & 26,2 & 22 & 14,2 & 36 & 54,5 \\
\hline De $2 \mathrm{~h} 30 \mathrm{~min}$ a $3 \mathrm{~h} 30 \mathrm{~min}$ & 65 & 29,4 & 49 & 31,6 & 16 & 24,2 \\
\hline De $3 \mathrm{~h} 30 \mathrm{~min}$ a $4 \mathrm{~h} 30 \mathrm{~min}$ & 59 & 26,7 & 47 & 30,3 & 12 & 18,2 \\
\hline Maior que $4 \mathrm{~h} 30 \mathrm{~min}$ & 39 & 17,6 & 37 & 23,9 & 2 & 3,0 \\
\hline Total & 221 & 100,0 & 155 & 100,0 & 66 & 100,0 \\
\hline
\end{tabular}

Nota: A análise estatística, comparando os dois ambulatórios, mostra os seguintes valores de $\mathrm{p}$ : A té $2 \mathrm{~h} 30 \mathrm{~min}$ :

$p=0,00001$; de $2 \mathrm{~h} 30 \mathrm{~min}$ a $3 \mathrm{~h} 30 \mathrm{~min}: \mathrm{p}=0,271$; de $3 \mathrm{~h} 30 \mathrm{~min}$ a $4 \mathrm{~h} 30 \mathrm{~min}: \mathrm{p}=0,061$; maior que $4 \mathrm{~h} 30 \mathrm{~min}: \mathrm{p}=0,0002$.

pessoas atendidas por serviços públicos de saúde - PACIENTES!

Ao compararem-se os dois ambulatórios, as diferenças surgem e são bem evidentes. No ambulatório geral, $14,2 \%$ da clientela esperam até duas horas e meia, e, na especialidade, o fato se dá com $54,5 \%$ ( $p=0,00001)$. Somente 3\% dos pacientes da especialidade esperam mais que quatro horas e meia, contra $23,9 \%$ do ambulatório geral ( $p=0,0002)$.

A média de espera foi respectivamente de quatro horas para o geral e de duas horas e quarenta minutos para a especialidade, diferença esta estatisticamente significante ( $p=$ 0,00001 ). A mediana foi de três horas e quarenta minutos para o primeiro e de duas horas e meia para o segundo. Assim, a clientela do ambulatório geral fica submetida a um tempo de espera realmente mais longo.

\section{Problemas no atendimento}

Relativos a qualquer aspecto da assistência à criança, dentro e fora do ambulatório de Pediatria, e relatados pelos responsáveis.

Dos usuários do ambulatório de Pediatria, $43,4 \%$ relataram algum tipo de problema envolvendo a assistência, sendo as diferenças encontradas entre os dois ambulatórios sem significação estatística.

Sugestões para a melhoria da assistência

Apontam para os problemas citados.

Embora $43,4 \%$ dos usuários relatem problemas, apenas $17,6 \%$ conseguem opinar sobre sugestões para a melhoria da atenção médica. As sugestões são mais freqüentes no ambulatório geral (21,3\%) do que no de especialidade $(9,1 \%)$, com $p=0,029$.

Ao serem questionados sobre os problemas ou fal has no atendimento do ambulatório de
Pediatria e solicitados a respeito de sugestões para a melhoria do mesmo, nota-se nitidamente uma grande tolerância dos usuários com relação a eles.

Os problemas citados abrangem desde questões externas ao HC, como dificuldades de acesso (longa distância, custeio do transporte) e de transporte (falta de adequação nos horários de ambulâncias e ônibus), até questões internas, as quais poderíamos sistematizar da seguinte maneira: área física (falta de informação visual dentro do $\mathrm{HC}$, dificultando a locomoção do paciente, falta de higiene no $\mathrm{HC}$ ); organização e filas (demora no atendimento, longas filas, excesso de pessoas para serem atendidas, remarcação de cirurgias várias vezes, necessidade de se chegar muito cedo pela manhã, carência de um alto-falante na recepção, extravio de exames e prontuários, presença de fumantes na sala de espera, não-organização dos prontuários por ordem de chegada); recursos humanos (relacionamento ruim entre funcionários e clientela, funcionários informam mal); consulta médica (médicos diferentes, atendimento realizado por estudantes, demora para a criança melhorar, morosidade na investigação do caso, prescrição de medicamentos caros, necessidade de um médico como referência para situações de intercorrência, falta de clareza nas explicações médicas); retaguarda de investigação (exames muito demorados); acesso à especialidade e procedimentos (dificuldade para conseguir consulta em especialistas e para imunoterapia, demora no agendamento de cirurgias, difícil acesso a psicólogo); fluxo e integração (retornos agendados com intervalos muito longos, necessidade de vir ao $\mathrm{HC}$ muitas vezes para consultar e realizar exames); infra-estrutura (falta de remédios).

Dentre os problemas acima, merecem destaque a demora no atendimento, citada em 
19\% das entrevistas, o atendimento realizado por médicos diferentes em cada consulta, presente em $6,8 \%$, e o interval o muito longo entre os retornos, relatado em $5 \%$.

As sugestões dadas pelos usuários, de acordo com sua visão do serviço, tentam solucionar os diversos problemas citados.

Os relatos dos pacientes apontam os mais diversos tipos de problemas enfrentados, desde aspectos da área física, organizacionais, de recursos humanos, fluxo, integração e infra-estrutura, evidenciando a lógica de fragmentação interna com que funcionam os serviços de saúde no Brasil, não sendo o HC-Unicamp uma exceção, obviamente.

Nogueira (1994) sugere a aplicação de princípios organizacionais que resguardem o conforto do usuário e aumentem a percepção dos profissionais sobre o sentido do conjunto dos atos, para enfrentar muitos dos problemas que foram aqui apresentados.

Podemos, deste modo, propor que a satisfação do usuário seja uma meta neste ambulatório e que seja avaliada sistematicamente sob a perspectiva de quem utiliza o serviço vivenciando os problemas citados, trazendo, portanto, críticas e sugestões concretas para a melhoria da qualidade do serviço prestado. Agendar com horários marcados, levando-se em consideração a procedência do paciente e a forma de transporte utilizado; diminuir ao máximo o número de retornos ao HC para a realização de investigação, procurando discutir com os diversos setores de retaguarda formas de integrar o atendimento ambulatorial com a realização de exames; tornar o espaço físico mais confortável e humanizado e melhorar a informação visual para que o paciente se localize melhor dentro do HC são algumas propostas de modificações organizacionais não muito complexas e que poderiam resultar numa melhora sensível da qualidade.

\section{Conclusões}

Os resultados desta pesquisa permitem-nos concluir que o ambulatório de Pediatria constitui-se num local privilegiado, por ser um serviço de referência dentro das propostas do Sistema Único de Saúde, que integra assistência e docência aliadas a toda uma retaguarda tecnológica que poucos serviços em nosso País possuem. Tal fato possibilita aos profissionais que nele trabal ham ter uma visão crítica sobre a atuação do conjunto da rede de saúde, uma vez que a ele chegam os pacientes que não puderam ter seus problemas de saúde resolvidos em outra instância, demonstrando o limite de capacidade dessa rede em ser resolutiva.

Os indicadores de qualidade da atenção ali prestada são de forma geral bastante satisfatórios, sendo a falta de vinculação entre médico e paciente e a lógica fragmentada de sua organização os principais problemas apontados. As soluções anteriormente propostas para enfrentá-los são relativamente simples, não requerem o investimento de verbas e certamente produzirão melhoria na qualidade da assistência.

Ao se colocarem os usuários dos serviços públicos em posição central, resgatando-se o caráter público destes, a construção de novas lógicas na sua organização deverá buscar formas mais humanizadas de compreender e atender às necessidades dos que os buscam, resgatando-se sua cidadania. 


\section{Referências}

AGUIRRE-GAS, H., 1990. Evaluación de la calidad de la atención médica. Expectativas de los pacientes y de los trabajadores de las unidades médicas. Salud Pública de México, 32:170-180.

BLUMENTHAL, D., 1996. Quality of health care: What is it? New England Journal of Medicine, 335:891894.

CAM PERO-CUENCA, L., 1990. Calidad de la relación pedagógica médico-paciente en consulta externa: el caso de Oaxaca, México. Salud Pública de México, 32:192-204.

CAM POS, G. W. S., 1992. Modelos de atenção em saúde pública: um modo mutante de fazer saúde. Saúde em Debate, 37:16-19.

CHAVEZ, M. R.; MARTINEZ-NARVAEZ, G.; CALVORIOS, J. M.; AGUIRRE-GAS, H.; ARANGO-ROJAS, R.; LARA-CARREÑO, R.; CAMINOS-CHAVEZ, M. I.; ORTIZ-SOLALINDE, G.; UGALDE-GRAJEDA, E. \& HERRERA-LASSO, F., 1990. Bases para la evaluación de la calidad de la atención en las unidades médicas del sector salud. Salud Pública de MéxiCO, 32:156-169.

DONABEDIAN, A., 1978. Evaluación y monitoreo de la calidad de los servicios de salud. In: Seminario de Investigación de Servicios de Salud (Consejo Nacional de Ciencia y Tecnologia - Secretaria de Salubridad y Asistência, org.), 2a ed., pp. 65-81, Queretaro: Memorial Queretaro.

DONABEDIAN, A., 1979. The quality of medical care: a concept in search of a definition. Journal of Family Practice, 2:277-84.

DONABEDIAN, A., 1988. Los Espacios de la Salud: Aspectos Fundamentales de la Organización de la Atención Médica. México: Fondo de Cultura Económica, Biblioteca de la Salud (Serie Clásicos de la Salud).

DONABEDIAN, A., 1992. Evaluación dela Calidad de la Atención Médica. Investigaciones sobre Servicios de Salud: Una Antología. Washington: Organización Panamericana de la Salud (Publicación Científica, 534).

ESCUDERO, P., 1989. Bases para una Propuesta de Instrumentación que Permita Evaluar Condiciones de Eficiencia y Capacidad Resolutiva de Servicios de Salud. Washington: Organización Panamericana de la Salud.

GEYNDT, W., 1986. Sinopsis de los planteamientos para evaluar la calidad de la atención al paciente. Salud Pública de México, 28:221-229.
GOMES, E. G., 1989. Marco Conceptual y Consideraciones Metodológicas Prel iminares Para el Desarrollo de un Protocolo de Investigación sobre Evaluación dela Calidad dela Atención dela Salud en un Grupo de Países Americanos. Washington: Organización Panamericana de la Salud.

HORWITZ, A., 1978. Por qué no evaluamos y por qué debemos hacerlo? Salud Pública de México, 20: 171-176.

JESSEE, W. F., 1990. Garantía de la calidad en los hospitales de Estados Unidos. Salud Pública de Mé xico, 32:131-137.

KUMATE, J. \& ISIBASIS, A., 1986. Investigación clínica y la calidad de la atención médica. Salud Pública de México, 28:318-23.

MAGALHÃES, L. B., 1983. Avaliação do Subprograma de Assi stência à Saúde da Criança em Dois Postos de Saúde de Londrina, Paraná. Dissertação de Mestrado, São Paulo: Faculdade de Saúde Pública, Universidade de São Paulo.

MORERA, R. G., 1981. Enfoque metodológico de la investigación aplicada en los servicios de salud. Educación Médica y Salud, 15:21-29.

NOGUEIRA, R. P., 1994. Perspectivas da Qualidadeem Saúde. Rio de Janeiro: Qualitymark Editora.

OSBORNE, C. E. \&THOMPSON, H., 1975. Criteria for evaluation of ambulatory child health care by chart audit: development and testing of a methodology. Pediatrics, 56:625-692.

RUELAS-BARAJAS, E. \& VIDAL-PINEDA, L. M., 1990. Unidad de garantía de calidad. Estrategia para asegurar niveles óptimos en la calidad de la atención médica. Salud Pública de México, 32:225231.

SOMM ERS, P., 1983. Participación activa de los consumidores en el sistema de prestación de servicios de salud: una evaluación de la satisfacción de los pacientes. Bol etin dela Oficina Sanitaria Panamericana, 94:54-75.

THIOLLENT, M. J. M., 1987. Crítica Metodológica, Investigação Social e Enquete Operária. 5a ed., São Paulo: Editora Polis.

VELÁZQUEZ, H. A., 1978. Desarrollo de instrumentos de medición para la evaluación de servicios médicos. In: Seminario de Investigación de Servicios deSalud (Consejo Nacional de Ciencia y Tecnologia - Secretaria de Salubridad y Asistência, org.), 2a ed., pp. 90-102, Queretaro: Memorial Queretaro. 